Research Article

\title{
Solving Bisymmetric Solution of a Class of Matrix Equations Based on Linear Saturated System Model Neural Network
}

\author{
Feng Zhang (iD \\ Department of Navigation, Anhui Communications Vocational and Technical College, Hefei 230051, China \\ Correspondence should be addressed to Feng Zhang; zf84061856@163.com
}

Received 26 March 2021; Accepted 10 July 2021; Published 20 July 2021

Academic Editor: Fu-Kwun Wang

Copyright (C) 2021 Feng Zhang. This is an open access article distributed under the Creative Commons Attribution License, which permits unrestricted use, distribution, and reproduction in any medium, provided the original work is properly cited.

\begin{abstract}
In order to solve the complicated process and low efficiency and low accuracy of solving a class of matrix equations, this paper introduces the linear saturated system model neural network architecture to solve the bisymmetric solution of a class of matrix equations. Firstly, a class of matrix equations is constructed to determine the key problems of solving the equations. Secondly, the linear saturated system model neural network structure is constructed to determine the characteristic parameters in the process of bisymmetric solution. Then, the matrix equations is solved by using backpropagation neural network topology. Finally, the class normalization is realized by using the objective function of bisymmetric solution, and the bisymmetric solution of a class of matrix equations is realized. In order to verify the solving effect of the method in this paper, three indexes (accuracy, correction accuracy, and solving time) are designed in the experiment. The experimental results show that the proposed method can effectively reduce the solving time, can improve the accuracy and correction effect of the bisymmetric solution, and has high practicability.
\end{abstract}

\section{Introduction}

In the process of mathematical development, matrix equations has a relatively important position and significance, and it can solve those abstract problems effectively [1]. In today's network era of big data, mathematics can effectively analyze people's consumption trends, predict the ratio of men and women in the population [2], and analyze the economic development trend of the whole city and even the world, which has great development value in the financial industry, industrial production, agricultural planting industry, and so on [2].

For this reason, relevant scholars have studied and verified the solving problem of a class of matrix equations and made some progress [3]. In reference [4], a least square bisymmetric solution method for a class of matrix equations is proposed and the bisymmetric solution of a class of matrix equations constructed by least square calculation is solved. The algorithm convergence was proved by iterative sequence. This method can effectively improve the algorithm convergence, but the solving speed of matrix equations is slow. In reference [5], it is proposed that a class of matrix equations solving method based on double iterative algorithm, according to the matrix series method, the inverse matrix of a class of nonlinear matrix equation is transformed equivalently. Then, the polynomial transformation of the matrix equations is realized by the Schur interpolation method, and the conjugate gradient method is used for iterative solution. According to Newton algorithm, the final matrix equations is solved by double iteration. This method can effectively avoid falling into the local optimal solution problem, but the solving accuracy of the matrix equations of the double iteration algorithm is poor. Liang et al. put forward an In-N-MCG (InexactNewton-MCG) algorithm for solving a class of matrix equations. The Newton method was used to reduce the dimension of the matrix equations, and submatrix constraint was used to optimize the range of effective solution set. Numerical examples were used to verify the effectiveness of bivariate Riccati method. This method can effectively improve the time of solving the equations, but the effect of equations solution correction is not good.

In order to solve the problems existing in the traditional methods, in this paper, a new method based on LSSM (linear saturated system model) neural network for solving a 
class of matrix equations is proposed. The specific research process of this paper is as follows:

(1) The first step is to analyze the background and research status of a class of matrix equations and determine the core research issues.

(2) Secondly, the LSSM neural network structure is constructed to obtain the characteristic parameters in the solution process of the bisymmetric solution.

(3) In the third step, BP (backpropagation) neural network topology is used to design the algorithm of bisymmetric solution.

(4) In the fourth step, the objective function value is used to calculate the bisymmetric solution of a class of matrix equations, and the bisymmetric solution of a class of matrix equations is solved.

(5) In the fifth step, the validity of the proposed method is verified by the experiments of solving accuracy, correcting accuracy, and solving time.

\section{Solving Matrix Equations of Linear Saturated System Model Neural Network}

In order to solve the problem of complexity and low efficiency of solving a class of matrix equations, this paper introduces LSSM neural network structure [6] to solve the bisymmetric solution of a class of matrix equations. In this section, a kind of matrix equations is constructed, and the key problems to solve the equations are determined. Secondly, the structure of LSSM neural network is constructed to determine the characteristic parameters in the process of bisymmetric solution [7].

2.1. Construction of Neural Network Structure. Traditional deep learning is a stack of multiple network layers, with the output of the previous layer serving as the input of the next layer. BP neural network is a typical structure of deep learning training multilayer network. RBM (restricted Boltzmann machine) [8] is stacked as DBN (deep Boltzmann machine) [9]. The data features are extracted by the first RBM. Next, it is entered into the next RBM and so on. Finally, the data features of the whole network are extracted, and the data are predicted and classified by adding an output layer to the network model [10].

The best way to avoid the network weight parameters falling into local optimum is to use RBM to initialize the network model. In this way, the initialization speed of the model can be improved; the backpropagation algorithm is used for supervised learning of the data [11]. In this process, the parameters of the model can be continuously optimized, and finally the expected model can be constructed.

It is assumed that there are $m$ network information nodes in the LSSM neural network; in order to determine the solution range of bisymmetric solution according to the parameter characteristics, in this paper, LSSM neural network is used to train the solution set of a class of matrix equations. Firstly, the initial vector is obtained by rbm 1 training, and then the initial vector is transferred to the hidden layer of LSSM neural network according to the conjugate gradient method; secondly, in order to reconstruct the input signal of the visual layer, in the process of feature extraction, it is necessary to extract the visual layer data randomly, and the processed input signal is transmitted to the hidden layer [12]. This process is repeated continuously to generate the weights of $\mathrm{rbm} 1$, so as to obtain the output data similar to the input data. The initial weight $r_{1}$ of a class of matrix equations is kept constant [13]. The hidden layer input data of the first RBM are substituted into the visual layer of the second RBM, and then the second RBM is trained. Before completing all RBM training, the above process is continued, and then a set of $r_{1}, r_{2}, \ldots, r_{m}$ weights can be obtained [14]. In order to reduce the training time of the model, the deep learning network is combined with the hierarchical training mechanism, and the maximum likelihood function learning method is used to train each layer of data [15].

The LSSM neural network structure is used to train the eigenvalues of matrix bisymmetric solution [16]. The multiple term transformation is used to extract the eigenvalues step by step. The nearest neighbor propagation algorithm is used to adjust the characteristic parameters of the matrix equations [17]. When $r_{1}, r_{2}, \ldots, r_{m}$ is set as the initial weight of the matrix equations, the objective function of the bisymmetric solution is obtained as follows:

$$
f(s)=-\sum_{m} \frac{r_{m}}{m} y_{i}^{T} \log y_{i}^{\prime}
$$

where $s=\left\{r_{1}, r_{2}, \ldots, r_{m}\right\}$ and $s$ are the weights of the bisymmetric solution of the matrix equations, $y_{i}$ is the label of the sample, and $y_{i}^{\prime}$ is the prediction characteristic parameter of the model. The samples with labeled information are input into LSSM neural network, and LSSM neural network can return the error to the whole network model. After adjusting the parameters of DBN at each layer, the expected value can be obtained.

2.2. Determination of Characteristic Parameters for Bisymmetric Solution. Suppose $J$ is used to represent a class of matrix, $J \in R^{m}$, and there are some constraints in the operation of this class of matrix equations. In this study, the constraint conditions are set as follows:

$$
J_{m n}=J_{m-2, n+i}, \quad m, n=1,2, \ldots, m .
$$

When a matrix satisfies the above constraints, it is called a central bisymmetric matrix. In this class of matrices, the $m$ order center bisymmetric matrix can be combined to write $Z^{m \times n}$. Suppose that the elements of this class of matrix satisfy

$$
Y(r)=J_{m n}+g(r)
$$

This class of matrix is called central antisymmetric matrix [18]. In this class of matrices, the $m$-order anticentral bisymmetric matrices can be combined to write $Z^{\prime m \times n}$. In this link, the following two problems will be solved, and the specific problems are shown as follows.

If the matrix of the research object is set as $K \in R^{n}$, $L \in R^{b}$, and $J \in Z^{\prime m \times n}$, then there is 


$$
|K+L|=Y(r) .
$$

Let $E_{n}$ be the set of solutions of the above formula and $E_{n} \in Z^{m \times n}$ be given to solve $E_{n}^{\prime} \in Z^{m \times n}$; then, there is

$$
\left|E_{n}^{\prime}-E_{0}\right|=\min _{J \in m}\left|E_{n}-E_{0}\right|
$$

According to the above formula, let $f_{i}$ denote the $i$-th column of a class of $m$-order unit matrix $g_{i}, i=1,2, \ldots, n$. Then, $g_{i}$ can be called the identity matrix of order $m$, with $g_{i}=f_{i}$.

According to the traditional method [19], the first condition of a class of matrix $J \in Z^{\prime m \times n}$ is $R_{i} * R_{i}=J[8,9]$. Secondly, if $J \in Z^{m \times n}$, then there is $R_{i}+J \in Z^{m \times n}$. By integrating the above constraints, the following formula can be obtained:

$$
E_{i}\left(R_{i}+J\right)+f(s)=g_{i} .
$$

The above formula is analyzed. If $K, L \in R^{n}, \alpha_{1} K+\alpha_{2} L$, then $\alpha_{1} K+\alpha_{2} L \in H^{\prime m \times n}$. The analysis of this formula shows that $H^{\prime m \times n}$ is the subspace of $R^{n}$. The results are as follows:

$$
R^{n}\left(\alpha_{1} K+\alpha_{2} L\right)=\alpha_{1} K R^{n}+\alpha_{2} L R_{i} .
$$

Through the above formula, the solving process of a class of central bisymmetric matrix equations is constrained, and this equations must have a central bisymmetric solution. The solution result is the least square solution of a class of matrix equations, that is, the solution of the above problem.

\section{Standard Calculation of Objective Function Value}

Based on the characteristic parameters in the process of bisymmetric solution determined above, the following BP neural network topology is used to solve the matrix equations. Class normalization is realized by using the objective function of bisymmetric solutions to realize the bisymmetric solutions of a class of matrix equations.

3.1. Construction of Neural Network. In order to solve a class of matrix equations, it is necessary to construct the topological structure of BP neural network [20]. The main way to train and optimize the network parameters is error backpropagation, which has strong adaptive processing ability [21]. The topological structure of BP neural network is shown in Figure 1.

In the feasible region of a class of matrix equations, the standard calculation of objective function value is carried out by combining the nonsmooth function algorithm, which improves the applicability of nonsmooth function [22]. Under the condition of constant initial boundary conditions, the joint features of a class of matrix equations features are linked and corresponding exchange samples are provided, so that differential crossover and mutation operators can be better collected and calculated [23]. In the process of calculation, it is easy for the phenomenon of partial bit exchange of crossover operator to appear, which needs to be replaced in order to solve the final results of a class of matrix

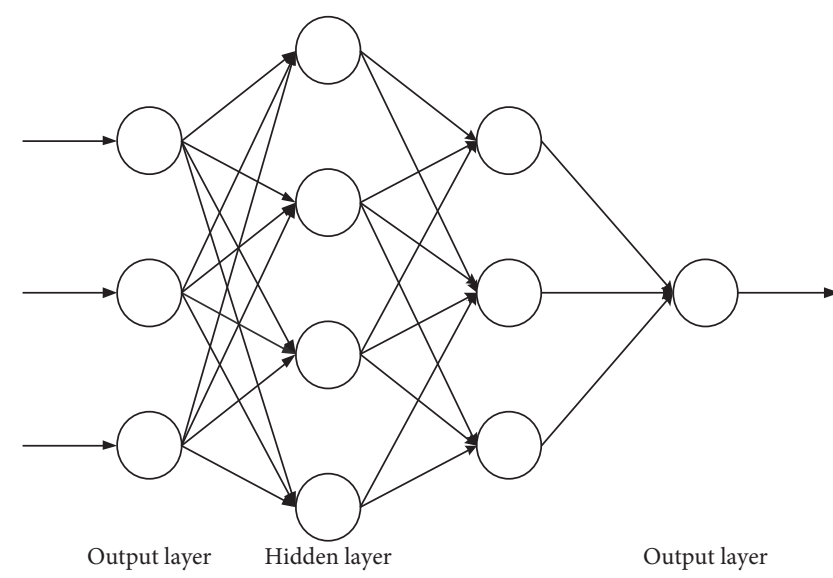

FIgUre 1: Topological structure of BP neural network.

equations [24]. In order to avoid the common problem of inertial rheological parameters in traditional methods, the characteristic variables and linear Galerkin method are combined to compare the derivative results to improve the calculation accuracy.

3.2. Modification of Optimal Solution for a Class of Matrix Equations. In a class of matrix equations, if the quasi-steady state of convergence variable is $f_{\text {in }}$, the characteristic value of dynamic information of inertial flow variable is $D_{\text {in }}$, and the approximate ratio of gradient inertial flow is $f_{\text {in }}$. In order to improve the effectiveness of the algorithm, the numerical model of singularity is established by using the inertia integral of the objective solving function of the bisymmetric solution of a class of matrix equations, and the nonsmooth characteristics of a class of matrix equations are constrained, so as to achieve better calculation accuracy, and the parameter characteristic constraint algorithm of a class of matrix equations is optimized.

$$
f(t)=v_{i}+D_{\text {in }} \sqrt{G_{t}},
$$

where $v_{i}$ is the independent variable of the inertial flow of a class of matrix equations, $G_{t}$ is the unknown parameter, and $m=m_{1}+m_{2}+\cdots+m_{n}$ is the constraint value.

Based on the characteristics of random variable function, the relationship between the unknown parameters and the simplicity of a class of matrix equations leads to infinite solutions of a class of matrix equations [24]. Therefore, it is necessary to set the fixed value conditions for the definite solutions. The iterative algorithm is used to realize the fast approximation and error control of the definite solution. By introducing multiple bisymmetric parameter features, the iteration is carried out, and the range of values is continuously reduced to reduce the error correction value of bisymmetric solution of a class of matrix equations. The specific algorithm is as follows:

$$
f_{i n}^{\prime}=G_{t} \varepsilon+\frac{\Delta g}{v_{i}} .
$$

Further combining with the limitation of the objective function, the range of the optimal solution is deduced, and the specific derivation principle is as follows: 
TABLE 1: Accuracy of bisymmetric solution under different methods.

\begin{tabular}{|c|c|c|c|c|}
\hline \multirow{2}{*}{ Iterations/time } & \multicolumn{4}{|c|}{ Accuracy of bisymmetric solution (\%) } \\
\hline & Least square method & Double iteration algorithm & In-N-MCG algorithm & Method of this paper \\
\hline 10 & 76 & 64 & 64 & 97 \\
\hline 20 & 73 & 34 & 38 & 94 \\
\hline 30 & 47 & 54 & 65 & 98 \\
\hline 40 & 66 & 57 & 57 & 96 \\
\hline 50 & 57 & 65 & 35 & 91 \\
\hline 60 & 72 & 68 & 57 & 95 \\
\hline 70 & 65 & 72 & 71 & 94 \\
\hline 80 & 62 & 46 & 68 & 99 \\
\hline 90 & 64 & 54 & 72 & 95 \\
\hline 100 & 65 & 43 & 53 & 96 \\
\hline Mean value & 64.7 & 55.7 & 58 & 95.5 \\
\hline
\end{tabular}

TABLE 2: Correction accuracy of bisymmetric solution under different methods.

\begin{tabular}{lcccc}
\hline \multirow{2}{*}{ Iterations/time } & \multicolumn{2}{c}{ Correction accuracy of bisymmetric solution (\%) } \\
& Least square method & Double iteration algorithm & In-N-MCG algorithm & Method of this paper \\
\hline 10 & 66 & 69 & 82 & 89 \\
20 & 65 & 50 & 65 & 95 \\
30 & 83 & 83 & 73 & 97 \\
40 & 54 & 74 & 42 & 92 \\
50 & 82 & 68 & 65 & 93 \\
60 & 75 & 43 & 83 & 89 \\
70 & 65 & 56 & 58 & 97 \\
80 & 56 & 43 & 43 & 94 \\
90 & 53 & 54 & 65.5 & 93 \\
100 & 64 & 67 & 69 \\
Mean value & 66.3 & 60.7 & & 93.6 \\
\hline
\end{tabular}

$$
\begin{aligned}
G_{t}:(x, \varepsilon, v) & \longrightarrow b(m):(x, i+q, f), \\
G_{t}^{\prime}:\left(x^{\prime}, \varepsilon^{\prime}, v^{\prime}\right) & \longrightarrow b(m):\left(x+f_{\mathrm{in}}, i+\Delta t, f+\Delta g\right) .
\end{aligned}
$$

Through the above process, the optimal solution of a class of matrix equations of the largest order can be obtained by multiple iterations effectively, and the accuracy of solving the results of the bisymmetric solution can be improved better.

\section{Experiment}

In order to verify the effectiveness of this method, this paper uses the least square method, double iterative algorithm, InN-MCG algorithm, and this method to predict the accuracy of concrete compressive strength. The specific experiments are as follows.

4.1. Accuracy of Bisymmetric Solution. In order to verify the solution effect of the bisymmetric solution under this method, the least square solution method, double iterative algorithm, In-N-MCG algorithm, and the method in this paper are used to compare the accuracy of solving the bisymmetric solutions. The results are shown in Table 1.

According to Table 1, for the three traditional methods, the matrix equations of this method can obtain more accurate bisymmetric solution, and the accuracy is as high as $94 \%$. The accuracy of matrix equations solution set is improved. When the number of iterations for a class of matrix equations is 80 , the accuracy of solving a class of matrix equations by this method can reach $99 \%$. The overall analysis shows that the average accuracy of the bisymmetric solution of a class of matrix equations is $95.5 \%$, which has higher accuracy of solving a class of matrix equations and higher application value.

4.2. Correction Accuracy of Bisymmetric Solution. In order to verify the correction effect of bisymmetric solution under this method, the least square method, double iteration algorithm, In-N-MCG algorithm, and this method are used to compare the correction accuracy of bisymmetric solution. The results are shown in Table 2.

According to Table 2, with the increase of the number of experimental iterations, the correction accuracy of our method has been maintained at a high level, with the lowest correction accuracy of $89 \%$ and the highest correction accuracy of $99 \%$. By analyzing Table 2 , it can be seen that the average correction accuracy of the proposed method is $93.6 \%$, which can effectively improve the correction accuracy of the bisymmetric solution.

4.3. The Solution Time of Bisymmetric Solution. In order to verify the efficiency of the bisymmetric solution under this method, the least square method, double iteration method, 
TABLE 3: The solution time of bisymmetric solution under different methods.

\begin{tabular}{|c|c|c|c|c|}
\hline \multirow{2}{*}{ Iterations/time } & \multicolumn{4}{|c|}{ Dissolution time/s } \\
\hline & Least square method & Double iteration algorithm & In-N-MCG algorithm & Method of this paper \\
\hline 10 & 66 & 55 & 66 & 2 \\
\hline 20 & 54 & 67 & 48 & 6 \\
\hline 30 & 68 & 84 & 56 & 5 \\
\hline 40 & 76 & 75 & 55 & 3 \\
\hline 50 & 65 & 57 & 49 & 7 \\
\hline 60 & 73 & 65 & 52 & 4 \\
\hline 70 & 65 & 72 & 63 & 8 \\
\hline 80 & 63 & 65 & 58 & 3 \\
\hline 90 & 62 & 63 & 55 & 5 \\
\hline 100 & 43 & 65 & 65 & 5 \\
\hline Mean value & 63.5 & 66.8 & 56.7 & 4.8 \\
\hline
\end{tabular}

In-N-MCG algorithm, and this method are used to compare the solution time of bisymmetric solution. The results are shown in Table 3.

By analyzing Table 3, it can be seen that different methods take different time to solve the bisymmetric solutions of a class of matrix equations. However, the efficiency of this method is much higher than that of the other three methods, less than one tenth of the other three methods, and only $4 \mathrm{~s}$.

According to the above experimental results, the solution accuracy of the proposed method is higher, and the accuracy is up to $94 \%$. In this method, the correction effect of bisymmetric solution is more ideal, and the solution time is shorter. However, due to the limitation of time and energy, there are still some deficiencies in this study. In the future, it is necessary to simplify the solving process of a kind of matrix equations to obtain higher solving efficiency.

\section{Conclusion}

In this paper, a method for solving bisymmetric solution of a class of matrix equations based on LSSM neural network is proposed. In order to verify the effectiveness of the method of solving bisymmetric solution of a class of matrix equations based on LSSM neural network, the accuracy, correction accuracy, and solution time of the bisymmetric solutions are tested.

The average accuracy of solving the bisymmetric solution of a class of matrix equations is as high as $95.5 \%$, and the average correction accuracy of solving the bisymmetric solution is as high as $93.6 \%$, and the average time of solving the bisymmetric solution is $4.8 \mathrm{~s}$. The method proposed in this paper has high accuracy for solving a class of matrix equations and can effectively shorten the time of solving the bisymmetric solution of a class of matrix equations and improve the correction rate of solving the bisymmetric solution.

In the future, this paper needs to simplify the solving process of a class of matrix equations in order to obtain higher solving efficiency.

\section{Data Availability}

The data used to support the findings of this study are available within the article.

\section{Conflicts of Interest}

The author declares that there are no conflicts of interest.

\section{References}

[1] M. Hajarian and A. T. Chronopoulos, "Least-squares partially bisymmetric solutions of coupled sylvester matrix equations accompanied by a prescribed submatrix constraint," Mathematical Methods in the Applied Sciences, vol. 12, no. 6, pp. 26-35, 2020.

[2] D. Guo, H. Tang, A. O. En et al., "Coefficient estimates for a class of $\mathrm{m}$-fold symmetric bi-univalent function defined by subordination," Communications in Mathematical Research, vol. 35, no. 1, pp. 57-64, 2019.

[3] R. Erfanifar, K. Sayevand, and H. Esmaeili, "A novel iterative method for the solution of a nonlinear matrix equations," Applied Numerical Mathematics, vol. 153, no. 4, pp. 1569$1580,2020$.

[4] S. Pakhira, S. Bose, and S. M. Hossein, "Solution of a class of nonlinear matrix equations," Bulletin of the Iranian Mathematical Society, vol. 15, no. 21, pp. 33-38, 2020.

[5] C. H. M. Kim, "Elementwise minimal nonnegative solutions for a class of nonlinear matrix equations," East Asian Journal on Applied Mathematics, vol. 9, no. 4, pp. 665-682, 2019.

[6] H. K. Nashine and S. Bose, "Solution of a class of crosscoupled nonlinear matrix equations," Applied Mathematics and Computation, vol. 43, no. 32, pp. 362-375, 2019.

[7] L. Lv, J. Chen, Z. Zhang et al., "A numerical solution of a class of periodic coupled matrix equations," Journal of the Franklin Institute, vol. 21, no. 34, pp. 32-45, 2020.

[8] A. Kittisopaporn, P. Chansangiam, and W. Lewkeeratiyutkul, "Convergence analysis of gradient-based iterative algorithms for a class of rectangular sylvester matrix equations based on banach contraction principle," Advances in Difference Equations, vol. 2021, no. 1, pp. 1-17, 2021.

[9] X. Xu, "Logarithmic upper bounds for weak solutions to a class of parabolic equations," Proceedings of the Royal Society of Edinburgh: Section A Mathematics, vol. 149, no. 6, pp. 1481-1491, 2019.

[10] Y. I. Vinogradov, D. B. Molchanov, and V. N. Bakulin, "Analytical matrix solutions of linear ordinary differential equations with constant coefficients," Journal of Physics: Conference Series, vol. 1392, no. 1, (5pp), Article ID 012077, 2019.

[11] M. Hajarian, "Partially doubly symmetric solutions of general Sylvester matrix equations," Transactions of the Institute of Measurement and Control, vol. 42, no. 3, pp. 503-517, 2020. 
[12] J. Y. Chang, R. Y. Chen, and C. C. Tsai, "Symmetric method of approximate particular solutions for solving certain partial differential equations," Engineering Analysis with Boundary Elements, vol. 119, no. 22, pp. 105-118, 2020.

[13] M. Soltani and B. Asgarian, "Exact stiffness matrices for lateral-torsional buckling of doubly symmetric tapered beams with axially varying material properties," Iranian Journal of Science and Technology-Transactions of Civil Engineering, vol. 21, no. 6, pp. 33-42, 2020.

[14] F. Yu and R. Fan, "Nonstandard bilinearization and interaction phenomenon for PT-symmetric coupled nonlocal nonlinear schrödinger equations," Applied Mathematics Letters, vol. 103, pp. 106-119, 2020.

[15] A. V. Ivashkevich, E. M. Ovsiyuk, V. V. Kisel, and V. M. Red'kov, "Spherical solutions of the wave equations for a spin $3 / 2$ particle," Doklady of the National Academy of Sciences of Belarus, vol. 63, no. 3, pp. 282-290, 2019.

[16] F. M. Sakar and A. Canbulat, "Inequalities on coefficients for certain classes of m-fold symmetric and bi-univalent functions equipped with faber polynomial," Turkish Journal of Mathematics, vol. 43, no. 1, pp. 293-300, 2019.

[17] M. A. Ramadan, N. M. El-shazly, and B. I. Selim, "Iterative algorithm for the reflexive solutions of the generalized Sylvester matrix equations," Journal of the Egyptian Mathematical Society, vol. 27, no. 1, pp. 27-32, 2019.

[18] Z. Diab, J. L. G. Guirao, and J. A. Vera, "A note on the periodic solutions for a class of third order differential equations," Symmetry, vol. 13, no. 1, pp. 33-36, 2020.

[19] K. V. Mamalyha and M. M. Osypchuk, "On single-layer potentials for a class of pseudo-differential equations related to linear transformations of a symmetric $\alpha$-stable stochastic process," Carpathian Mathematical Publications, vol. 11, no. 2, pp. 34-38, 2019.

[20] Y. N. Wu, M. L. Zeng, and M. L. Zeng, "On ADMM-based methods for solving the nearness symmetric solution of the system of matrix equations $\mathrm{A} 1 \mathrm{XB} 1=\mathrm{C} 1$ and $\mathrm{A} 2 \mathrm{XB} 2=\mathrm{C} 2$," Journal of Applied Analysis \& Computation, vol. 11, no. 1, pp. 227-241, 2021.

[21] J. Tommasi and G. Palmiotti, "Verification of iterative matrix solutions for multipoint kinetics equations," Annals of $\mathrm{Nu}$ clear Energy, vol. 124, pp. 357-371, 2019.

[22] S. F. Yuan, M. Z. Li, and Y. Tian, "Numerical algorithms for solving the least squares symmetric problem of matrix equations $\mathrm{AXB}+\mathrm{CXD}=\mathrm{E}$," Filomat, vol. 33, no. 6 , pp. 1649-1658, 2019.

[23] E. Prasad, "Divergent solutions of linear programming problem (LPP) and system of linear equations (SLE) for a particular coefficient matrix," in Proceedings of the 1st International Conference on Mathematical Techniques and Applications: ICMTA2020, Chennai, India, November 2020.

[24] L. Lv, S. Tang, and L. Zhang, "Parametric solutions to generalized periodic sylvester bimatrix equations," Journal of the Franklin Institute, vol. 357, no. 6, pp. 54-63, 2020. 